\title{
Learning by Modeling (LbM): Understanding Complex Systems by Articulating Structures, Behaviors, and Functions
}

\author{
Kamel Hashem \\ Department of Learning Science, School \\ of Educational Sciences \\ Al-Quds University \\ Jerusalem, Palestine
}

\author{
David Mioduser \\ Department of Education in Math Science \\ and Technology, School of Education \\ Tel-Aviv University \\ Tel-Aviv, Israel
}

\begin{abstract}
Understanding the behavior of complex systems has become a focal issue for scientists in a wide range of disciplines. Making sense of a complex system should require that a student construct a network of concepts and principles about the learning complex phenomena. This paper describes part of a project about Learning-by-Modeling (LbM). Many features of complex systems make it difficult for students to develop deep understanding. Previous research indicates that involvement with modeling scientific phenomena and complex systems can play a powerful role in science learning. Some researchers argue with this view indicating that models and modeling do not contribute to understanding complexity concepts, since these increases the cognitive load on students. In this study we investigated the effect of different modes of involvement in exploring scientific phenomena using computer simulation tools, on students' mental model from the perspective of structure, behaviour and function. Quantitative and qualitative methods are used to report about 121 freshmen students that engaged in participatory simulations about complex phenomena, showing emergent, self-organized and decentralized patterns. Results show that LbM plays a major role in students' concept formation about complexity concepts.
\end{abstract}

Keywords-learning by modeling; simulation; complexity; mental models; educational technology

\section{INTRODUCTION}

Students' approach to complex dynamic systems and their mental models utilized to construct knowledge, play a powerful role in what students learn. The idea of complexity is increasingly becoming an integral part in learning natural and social sciences, where learning is understood to be more like practice of science [1]. Inquiry-based science, developing skills for systems thinking and adopting collaborative learning in science classes are all examples of that focus.

Students' perception when learning about complex systems is greatly aided by interactive simulations and models. Research indicates that learning through observation do not necessarily lead to strong intuitions or deep understanding of systems [2]. For example people observed bird flocks for thousands of years before anyone suggested that flocks are leader-less, and people participate in traffic jams without much understanding of what cause the jams, such phenomena may be regarded as complex systems. Observation and participation are not enough; people need a richer sense of involvement with systems in order to understand them [3], [4], [5], [6], [7], [8],
[9]. Modeling can provide students with the power to understand and explore systems that were previously difficult to trace and predict their behavior, new techniques that help to learn important concepts on complex systems, to generate relevant questions, theories and hypothesis about phenomena, and to build and run models related to their theories [10], [11], [12], [13], [14].

Emergent complex phenomena are considered to be difficult to understand [15], [16]. Despite the utilization of new learning approaches with models, students experience difficulties in learning concepts relevant to understanding complex systems currently taught in existing science courses student thinking may be counter-intuitive or might conflict with the scientific models, and the learning ideas concerning emergence or stochastic processes are difficult because of difference with teleological beliefs, where students tend to think of systems having centralized control [14], [15], [16], [17]. Hmelo-Silver and Pfeffer (2004) argue that the characteristics of complex systems make them difficult to understand, since they are comprised of multiple levels of organization that often depend on local interactions (the causes and effects are not obviously related); also it requires that students should construct a network of concepts and principles about the phenomena with complexity and their interrelationships [14], [16], [18].

Some researchers argue that modeling did not contribute a lot in understanding complexity since it increases the cognitive load on students (see [19]). This study focused on the effect of different modes of involvement in exploring scientific phenomena using computer modeling tools, on students' mental model from the perspective of the system structure, system behavior and function. It is part of a more comprehensive study pursuing the goals: (1) to study the role of modeling in the learning process of complexity and complex systems in the natural and artificial worlds; (2) to examine the contribution of different modes of involvement in the modeling process (e.g., observation and explanation, intervention and manipulation, programming and development) to the students' understanding of complexity; (3) to examine the effect of the level of complexity and properties (e.g., emergence, selforganized ...) of the systems being manipulated on the student's learning; and (4) to study the evolution in time of the 
TABLE I.

CATEGORIZATION OF CSMM ACROSS SBF CONCEPTS

\begin{tabular}{|l|l|}
\hline Mental Model & Questions \\
\hline Structure & $\begin{array}{l}\text { Describe what you see in detail (number of agents, how do agents behave before they are part of the system, system } \\
\text { environment)? }\end{array}$ \\
\hline Function & $\begin{array}{l}\text { Who/what initiates the formation of the system? } \\
\text { Are there feedback loops within the system? } \\
\text { Do they amplify or control the outcome? } \\
\text { How do agents behave before they are part of the system? } \\
\text { Is the same outcome will be achieved each time the system form? } \\
\text { How would the system respond to environmental change, explain why? }\end{array}$ \\
\hline Behaviour & $\begin{array}{l}\text { Is there movement of the agents within the system? } \\
\text { How would you design such system/explain its behavior? } \\
\text { Is there a difference between agents and system? } \\
\text { What draws the system together? }\end{array}$ \\
\hline
\end{tabular}

students' mental models of complexity as a function of the different variables (e.g., modes of involvement; level of complexity) of the system under study.

\section{METHOD}

\section{A. Subjects}

Participants are 121 undergraduate students (ages ranging from 18 to 20 years old) from the science department at AlQuds University in Jerusalem, divided into four groups by the kind of involvement in working with models: observation, exploration, manipulation, and model-development modes. All students attended a two hours introduction lesson to the NetLogo environment. The students were selected based on their scientific background, all have done the tawjehi exam as required by the ministry of education for the scientific track, and they are all studying first year compulsory science courses in the faculty of science.

\section{B. Research instruments}

(a) The learning environment comprising two components: (1) NetLogo, a specialized program developed at Northwestern University for agent-based modeling and for learning and understanding complex systems, "NetLogo is a multi-agent programming language and modeling environment for simulating natural and social phenomena. It is particularly well suited for modeling complex systems evolving over time. Modelers can give instructions to hundreds or thousands of independent "agents" all operating concurrently. This makes it possible to explore connections between micro-level behaviors of individuals and macro-level patterns that emerge from their interactions, it enables users to open simulations and "play" with them, exploring their behavior under various conditions. NetLogo is also an authoring environment that is simple

TABLE II. CATEGORIZATION OF CONCEPTS RELATED COMPLEX SYSTEMS MENTAL MODELS (CSMM)

\begin{tabular}{|c|c|c|}
\hline Parameters & $\begin{array}{l}\text { Clockwork component coding } \\
\text { (reductive) }\end{array}$ & $\begin{array}{l}\text { Complexity component coding } \\
\text { (non-reductive) }\end{array}$ \\
\hline $\begin{array}{l}\text { System control } \\
\text { 1. Who/what initiates the formation of the } \\
\text { system? }\end{array}$ & $\begin{array}{c}\text { Centralized } \\
\text { Order/control come from outside. }\end{array}$ & $\begin{array}{l}\text { Decentralized } \\
\text { Agents' actions are independent of each other; they } \\
\text { operate under the same rules. }\end{array}$ \\
\hline $\begin{array}{l}\text { Action effects } \\
\text { 1. Are there feedback loops within the system? } \\
\text { 2. Do they amplify or control the outcome? }\end{array}$ & $\begin{array}{l}\text { Linear } \\
\text { One thing leads to another, direct link } \\
\text { between cause and effect. }\end{array}$ & $\begin{array}{l}\text { Non-linear } \\
\text { Positive feedback can exhibit exponential results. } \\
\text { Effects are not straightforward functions of causes }\end{array}$ \\
\hline $\begin{array}{l}\text { Agents' action } \\
\text { 1. How do agents behave before they are part } \\
\text { of the system? }\end{array}$ & $\begin{array}{l}\text { Predictable } \\
\text { Agents' actions are predictable; there is no } \\
\text { mention of randomness or chance in their } \\
\text { action. }\end{array}$ & $\begin{array}{l}\text { Random } \\
\text { 1. Agents appear to act in random independent } \\
\text { fashion. } \\
\text { 2. Randomness allows for variability and variety } \\
\text { within the system. }\end{array}$ \\
\hline $\begin{array}{l}\text { Underlying causes } \\
\text { 1. Is the same outcome will be achieved each } \\
\text { time the system form? } \\
\text { 2. How would the system respond to } \\
\text { environmental change, explain why? }\end{array}$ & End point is predictable (teleologic) & $\begin{array}{l}\text { Probabilistic causes (stochastic) } \\
\text { 1. The system organizes itself based on agents } \\
\text { interactions, the resulting structure is never } \\
\text { certain. } \\
\text { 2. The system maintains its coherence/structure. }\end{array}$ \\
\hline
\end{tabular}


enough to enable students and researchers to create their own models, even if they are not professional programmers", in a Netlogo environment, students come to understand these concepts and laws through a process of exploration and inquiry by investigating and controlling the behavior of thousands of graphical "agents". By interactively exploring the relationship between the agents' rules of behavior and the patterns that emerge as a result of these rules, students are able to "debug" misconceptions that are generated by confusing their understandings of micro and macro level interactions. Typically, in curricula using multi-agent modeling, students begin by exploring the behavior of pre-built simulations designed to focus on some target concepts. They make predictions about the behavior of the model under varying model parameters then test their predictions by exploring model outcomes as they change 'sliders' in a simple graphical user interface (see figure below). The core of every NetLogo model is the interface window. Typically, the interface contains a graphics window, a plotting window and several variables in the form of sliders and buttons that the student can manipulate. It is here that students can observe directly the interaction between the micro- and macro-levels". [20: 1-2].

and (2) tasks and activities in which students run NetLogo models and are requested to perform tasks with the models, and

(b) Data collection tools included: (1) pre-test comprising general background and demographic information (e.g., major area, gender) and four questions dealing with complexity concepts such as emergence, self-organization and decentralization; (2) structured observation and data forms; (3) mental model worksheet focusing on students' complexsystem-mental-model (CSMM), completed by the end of each activity.

\section{Procedure}

The study was carried in four stages: (a) Pre-test, (b) Treatment in four different modes. Observation group: in 2 sessions of 90-minutes students were introduced to two models (two levels of complexity), requested to observe agents interactions, and to complete the CSMM worksheet. Exploration group: in 2 sessions of 90-minutes students were introduced to two models (two levels of complexity), were given an initial set of conditions for the system followed by a final set of conditions in which one or two parameters were changed (e.g., change in a variable-slider or a switch) while the others remained constant. After each model students were interviewed and requested to complete the worksheet. Manipulation group: in 2 sessions of 90-minutes students were introduced to two models (two levels of complexity) using NetLogo. They were asked about how the system would change if the system variables were altered, and even allowed to use NetLogo commands. The students then manipulated the system variables according to the interviewer's questions, explained their observations of the system's behavior and compared these with their initial predictions. After each model students completed the worksheet. Development and Design group: students were introduced to the Netlogo programming environment (48 hours), in order to have the ability to construct the learning models. After each model students were asked to complete the worksheet, (c) Interview after treatment: students were interviewed for their CSMM, all responses were audio taped and (d) Post-test: (same as pre-test).

\section{Scoring}

First coding scheme was based on the distinction between a system's structure, behavior, and function (SBF) (see Table I). Structure refers to elements of a system and their configuration (e.g., agents, environment, and interaction between them); behavior refers to how systems achieve their purpose through the interactions or of its agents and Function refers to the purpose of the agents in a given system. The second coding scheme was based on the categorization defined by Jacobson (2001), shown in Table II.

Students' answers were coded as non-reductive if these referred to a complexity-related matter (i.e. the whole is greater than the parts). Otherwise, if there was evidence of a stepwise approach to the explanation, the answer was coded as reductive (i.e. agents act in isolation). Jacobson (2001) refer to the reductive way of thinking as "deterministic and clockwork order".

\section{RESUlts}

\section{A. Quantitative analysis}

Student's responses were coded in terms of the various types of component beliefs reflected in their answers, for the pre-post test results showed an increase in students' understanding of complexity concepts in all the four groups (observation, exploration, manipulation, and development and design) (see Hashem and Mioduser, 2011).

TABLE III. STUDENTS' CSMM (FREQUENCIES AND PERCENTAGES) ACROSS SBF CONCEPTS FOR THE DIFFERENT MODES OF INVOLVEMENT (** P<0.01)

\begin{tabular}{|c|c|c|c|c|c|c|c|}
\hline \multirow{3}{*}{ Modes of Involvement } & \multirow{3}{*}{$(\mathrm{N})$} & \multicolumn{2}{|c|}{$\begin{array}{c}\text { Structure } * * \\
\text { Concept presence }\end{array}$} & \multicolumn{2}{|c|}{ Function $* *$} & \multicolumn{2}{|c|}{ Behaviour ** } \\
\hline & & No & Yes & Clockwork & Complex & Clockwork & Complex \\
\hline & & Frq $(\%)$ & Frq $(\%)$ & Frq $(\%)$ & Frq $(\%)$ & Frq $(\%)$ & Frq (\%) \\
\hline Observation & 58 & $32(18)$ & $142(81)$ & $162(55)$ & $126(43)$ & $118(67)$ & $40(22)$ \\
\hline Exploration & 66 & $11(5)$ & $187(94)$ & $143(43)$ & $185(56)$ & $117(59)$ & 75 (37) \\
\hline Manipulation & 56 & $8(4)$ & $160(95)$ & $103(36)$ & $173(61)$ & $91(54)$ & $76(45)$ \\
\hline Design & 62 & $11(5)$ & $175(94)$ & $90(29)$ & $217(70)$ & $76(40)$ & $101(54)$ \\
\hline
\end{tabular}


TABLE IV. : STUDENTS' MENTAL CONCEPTS ACROSS SBF CONCEPTS VS. MODES OF INVOLVEMENT $(* \mathrm{P}<0.05, * * \mathrm{P}<0.01)$ TABLE V.

\begin{tabular}{|c|c|c|c|c|c|c|}
\hline \multirow{3}{*}{$\begin{array}{c}\text { Complexity } \\
\text { Level }\end{array}$} & \multirow{3}{*}{ MM concept } & \multirow{3}{*}{ Response } & \multicolumn{4}{|c|}{ Group } \\
\hline & & & Observation & Exploration & Manipulation & Design \\
\hline & & & $\operatorname{Frq}(\%)$ & $\operatorname{Frq}(\%)$ & $\operatorname{Frq}(\%)$ & $\operatorname{Frq}(\%)$ \\
\hline \multirow{6}{*}{ Complicated } & \multirow{2}{*}{$\begin{array}{l}\text { Structure } * * \text { - } \\
\text { concept presence }\end{array}$} & No & $15(17.2)$ & $4(4)$ & $3(3.6)$ & $6(6.5)$ \\
\hline & & Yes & $72(82.5)$ & $95(96)$ & $81(96.4)$ & $87(93.5)$ \\
\hline & \multirow{2}{*}{ Function $* *$} & Clockwork & $79(55.2)$ & $78(47.6)$ & $43(31.6)$ & $45(29.4)$ \\
\hline & & Complex & $64(44.8)$ & $86(52.4)$ & $93(68.4)$ & $108(70.6)$ \\
\hline & \multirow{2}{*}{ Behavior $* *$} & Clockwork & $59(72.8)$ & $63(67)$ & $44(53)$ & $42(47.7)$ \\
\hline & & Complex & $22(27.2)$ & $31(33)$ & $39(47)$ & $46(52.3)$ \\
\hline \multirow{6}{*}{ Complex } & \multirow{2}{*}{$\begin{array}{l}\text { Structure } * * \text { - } \\
\text { concept presence }\end{array}$} & No & $17(19.5)$ & $7(7.1)$ & $5(6)$ & $5(5.4)$ \\
\hline & & Yes & $70(80.5)$ & $92(92.9)$ & $79(94)$ & 88 (94.6) \\
\hline & \multirow{2}{*}{ Function $* *$} & Clockwork & $83(57.2)$ & $65(39.6)$ & $60(42.9)$ & $45(29.2)$ \\
\hline & & Complex & $62(42.8)$ & $99(60.4)$ & $80(57.1)$ & $109(70.8)$ \\
\hline & \multirow{2}{*}{ Behavior $* *$} & Clockwork & $59(76.6)$ & $54(55.1)$ & $47(56)$ & $34(38.2)$ \\
\hline & & Complex & $18(23.4)$ & 44 (44.9) & $37(44)$ & $55(61.8)$ \\
\hline
\end{tabular}

As expected, students in the development and design group identified more concepts across the structure, function and behavior (SBF) framework than the other groups. A general log-linear analysis was conducted to examine the differences between the groups in their representation on structures, behaviors, and function, showing significant interaction between the modes of involvement and SBF concepts $(\chi 2(\mathrm{df}=$ 25) $=100.860, \mathrm{p}<0.01)$.

Table III presents the frequencies and percentages of the students' responses on the CSMM across the SBF concepts for the four groups (observation, exploration, manipulation and design). A chi-square test was done to check these frequencies for significance; results show a significant relationship between the different modes of involvement and the CSMM across the SBF concepts as follows: (a) Students largely identified a target list of questions regarding structure in the complex system mental model $(\mathrm{CSMM})(\chi 2(\mathrm{df}=3)=28.588, \mathrm{p}<.01)$, in examining the observed cell frequencies from Table III, it shows that that the manipulation group got the highest frequency $(95 \%)$ in identifying the concepts regarding system, followed by the design group and the exploration group (94\%) and finally the observation group (81\%), (b) Students largely favor to choose the clockwork model when they were asked questions regarding system functioning $(\chi 2(\mathrm{df}=3)=47.151, \mathrm{p}$ $<.01$ ), in examining the observed cell frequencies from Table III, the observation group showed the highest frequency $(55 \%)$ in favoring the clockwork model, on the other hand the design group showed high response in choosing the complex model on system functioning $(70 \%)$ followed by the manipulation group $(61 \%)$ followed by the exploration group $(56 \%)$ and finally the observation group (43\%), and (c) Students largely favor to choose the clockwork model when they were asked questions regarding system behavior $(\chi 2(\mathrm{df}=3)=36.043, \mathrm{p}<.01)$, in examining the observed cell frequencies from Table III, the observation group showed the highest frequency (67\%) in favoring the clockwork model followed by the exploration group (59\%) followed by the manipulation group (54\%) and finally the design group (40\%), on the other hand the design group showed the highest response in choosing the complex model on system behavior (54\%) followed by the manipulation group $(45 \%)$ followed by the exploration group (37\%) and finally the observation group (22\%).

The awareness regarding complex system mental model (CSMM) that was mentioned in Table II, can be seen across the different mental concepts: structure, function and behavior (SBF) that was mentioned in Table I with different complexity levels in Table IV, the different groups showed a significant interaction with the CSMM across the SBF concepts while interacting with models of different complexity levels. A general log-linear analysis was conducted using SPSS software to examine the differences between complexity levels (complicated and complex) and students perception on the CSMM across SBF concepts showing significant relationship $(\chi 2(\mathrm{df}=25)=68.769, \mathrm{p}<0.01)$ for complicated model and $(\chi 2(\mathrm{df}=25)=65.517, \mathrm{p}<0.01)$ for complex model.

Table IV and Figure 1 presents the frequencies and percentages of the students' responses on the CSMM across SBF concepts with different complexity levels for the four groups (observation, exploration, manipulation and design). A chi-square tests was done to check these frequencies for significance, results show a significant relationship between the different complexity levels and the different complexity concepts as follows:

(1) For the complicated model: (a) Students largely identified a target list of questions regarding structure in the model under study $(\chi 2(\mathrm{df}=3)=15.204, \mathrm{p}<.01)$, in examining the observed cell frequencies from Table IV, it shows that that the manipulation group got the highest frequency $(96.4 \%)$ in identifying the model structure, followed 

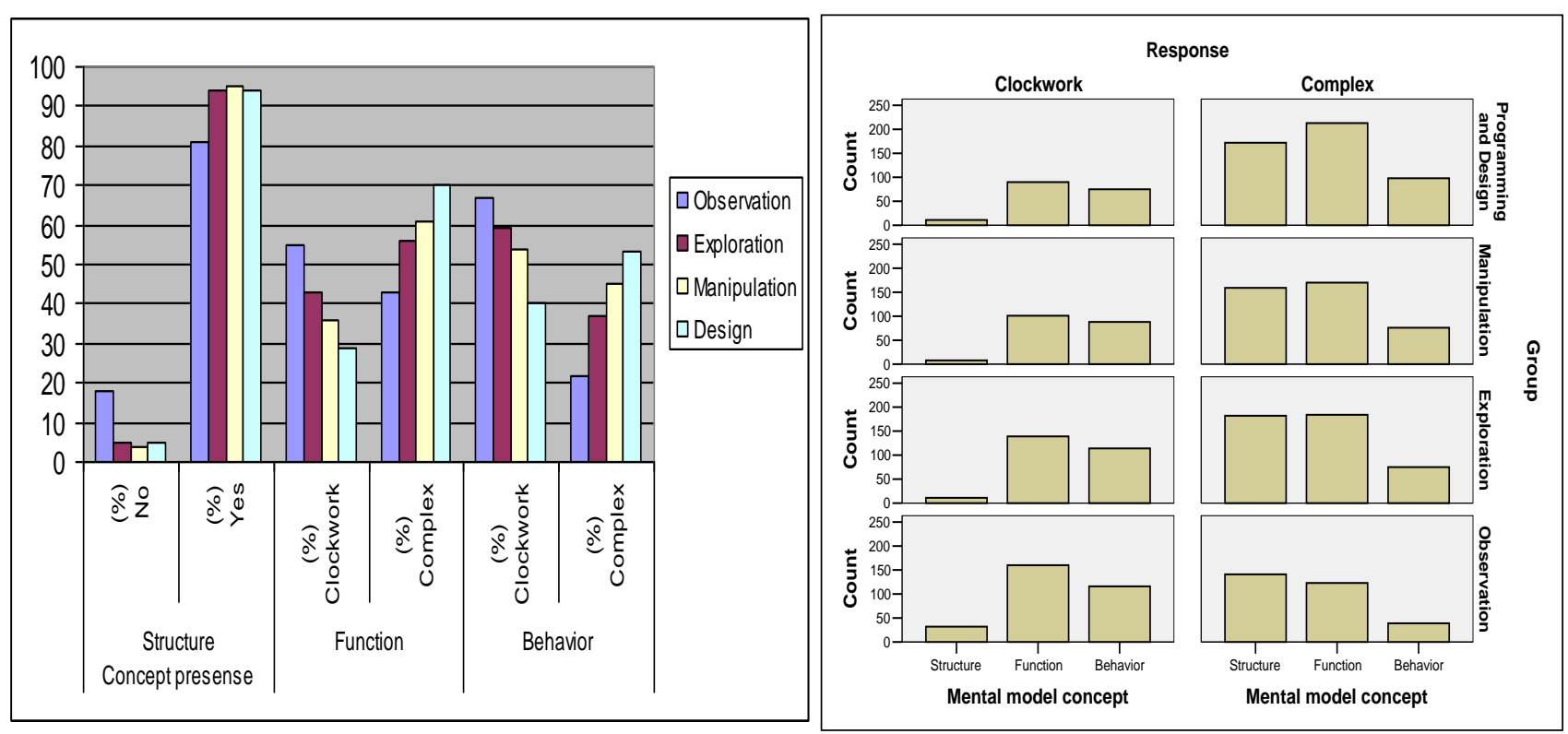

Fig.1. STUDENTS' CSMM ACROSS THE SBF LAYERS FOR THE DIFFERENT LEVELS OF INVOLVEMENT

by the exploration group (96\%) followed by the design group $(93.5 \%)$ and finally the observation group $(82.5 \%)$, (b) Students largely favor to choose the clockwork model when they were asked questions regarding system functioning $(\chi 2 \mathrm{df}$ $=3)=28.331, \mathrm{p}<.01)$, in examining the observed cell frequencies from Table IV, the observation group showed the highest frequency $(55.2 \%)$ in favoring the clockwork model, on the other hand the design group showed high response in choosing the complex model on system functioning (70.6\%) followed by the manipulation group (68.4\%) followed by the exploration group (52.4\%) and finally the observation group (44.8\%), and (c) Students largely favor to choose the clockwork model when they were asked questions regarding system behavior $(\chi 2(\mathrm{df}=3)=14.718, \mathrm{p}<.01)$, in examining the observed cell frequencies from Table IV, the observation group showed the highest frequency (72.8\%) in favoring the clockwork model followed by the exploration group (67\%) followed by the manipulation group (53\%) and finally the design group (47.7\%), on the other hand the design group showed the highest response in choosing the complex model on system behavior (52.3\%) followed by the manipulation group (47\%) followed by the exploration group (33\%) and finally the observation group (27.2\%), and (2) For the complex model: (a) Students largely identified a target list of questions regarding structure in the model under study $(\chi 2(\mathrm{df}=3)=14.120, \mathrm{p}<$ .01 ), in examining the observed cell frequencies from Table IV, it shows that that the design group got the highest frequency (94.6\%) in identifying the model structure, followed by the manipulation group (94\%) followed by the exploration group $(92.9 \%)$ and finally the observation group $(80.5 \%)$, (b) Students largely favor to choose the clockwork model when they were asked questions regarding system functioning $(\chi 2$ (df $=3)=24.577, \mathrm{p}<.01$ ), in examining the observed cell frequencies from Table IV, the observation group showed the highest frequency (57.2\%) in favoring the clockwork model, on the other hand the design group showed high response in choosing the complex model on system functioning (70.8\%) followed by the exploration group (60.4\%) followed by the manipulation group (57.1\%) and finally the observation group (42.8\%), and (c) Students largely favor to choose the clockwork model when they were asked questions regarding system behavior $\left(\chi^{2}(\mathrm{df}=3)=24.726, \mathrm{p}<.01\right)$, in examining the observed cell frequencies from Table IV, the observation group showed the highest frequency $(76.6 \%)$ in favoring the clockwork model followed by the manipulation group (56\%) followed by the exploration group (55.1\%) and finally the design group (38.2\%), on the other hand the design group showed the highest response in choosing the complex model on system behavior (61.8\%) followed by the exploration group (44.9\%) followed by the manipulation group (44\%) and finally the observation group (23.4\%).

\section{B. Qualitative analysis}

An examination of students' responses indicated additional qualitative differences between the different modes of involvement. The programming and design group provided more elaborate responses as well as demonstrating more understanding across the SBF concepts followed by the manipulation group followed by the exploration group and finally the observation group, this was evident in their answers. All the groups have identified the various system structures, but on the behavioral and functional level the programming and design group have discussed in more details. For example, in a description for the traffic jam model, one of the students in the design group said:

....The system consists of a number of cars that are driving in different velocities along the street.... The traffic jam occurs when we have an increase in the number of cars, specially the 
number of the private ones, and there is a feedback loop since the movement of each car is affected by the car in the front and in back.... The system draws together because of the restricted movement for the cars and all behavior looks semi-organized, where the elements of the system work in consistent to perform the target objective....

In this example the student mentions the system structure consisting of (cars, street) and continues to discuss the function of the cars and how traffic jam occurs because of the number of cars (non-reductive), and the system achieve its purpose through the restricted interactions between the cars and all behavior looks semi-organized (non-reductive). A student from the observation group responded to the same instructions with the following:

.... The system consists of a group of cars and street all the cars are blue; only one is red.... one of the things that might impede the flow of traffic is the traffic lights or an accident.... No traces for feedback since the cars are going at same speed...

This student mentioned numerous structures (cars, street, and agents color), she describes the functionality of the system in a reductive way (no feedback loops, cars are going at same speed) and did not offer additional behavioral information. Integrated students responses were also evident in the interviews where students were asked about the traffic jam formation and how it occurs? For example in a response to this question one of the students in the observation group noted:

S: "First of all you might have an accident in the road or the road is not good to let drivers pass in a regular way..."

I: "ok, suppose we have no accidents and the road is good, is there any chance to a have a traffic jam?"

S: "Yes, let's say if we have a traffic light..."

In this answer, the student's response has been coded as 'reductive or clockwork' because it referred to a centralized control and deterministic single causality (i.e. the references to 'accidents, road is not good, and traffic light').

Once again, students in the design group include more structural, functional and behavioral non-reductive responses in their answers followed by the manipulation group followed by the exploration group and finally the observation group, most of the groups indicated high responses in identification of the various system structures for both complicated and complex systems.

\section{DISCUSSION AND CONCLUSION}

Results showed that learning about complicated systems is not easier than learning about complex system with all groups. One of the reasons may relate to the specific model they interacted with.

Some students found it difficult trying to understand the concept of emergence, when they were involved with the complicated model "chemical equilibrium". In the task, they focused on the actions of molecules at the micro level (i.e., how molecules move, interact...etc.), rather than noticing the collective interactions of all the molecules. This means that they did not focus on the sum of all interactions of the individual molecules across time. They aknowledged that these reach the equilibrium state from observing the statistics regarding number of interacting and yeilding molecules.

When involved with the complicated model, students in the manipulation group showed elaboration and understanding in terms of the approach unveiled (e.g., "clockwork" vs. "complex") for the concepts of emergence and selforganization.

In contrast, when working on the complicated model, the exploration group showed an elaboration and understanding on "the way that systems are governed". Their description was that the actions of the actors are not directed towards any goal and molecules A and B don't have to interact or achieve an equilibrium, they simply move around and collide randomly [2].

Learning by modeling provided the base for promoting doing with reflection and for helping students make connections to the world around them [6]. The groups' differences unveil which complexity level was better understood by the students. We can say that students involvement with systems with complexity level of 'complex' have elaborated and understood more complex systems concepts while interacting with models than the students who were involved with systems with complexity level of 'complicated'.

Ultimately this article reports on a study about the interaction between modes of learning with computer modeling tool and the understanding of complexity concepts, there are many systems concepts that we never directly experience or that violate our intuitions and challenges of our cognitive and metacognitive resources. The implementation of such an instructional approach in the curriculum would have many benefits for learners, such as interdisciplinary learning allowing to see common patterns across traditionally separate fields, new ways of thinking (systems thinking and decentralized thinking), exploration of tools to think with, and construction of models linking between local causes and global behavior.

By introducing this new perspective (LbM) using computer modeling for learning complexity and emergent phenomena, science learning will be more motivational and truthful, more inclusive and accessible to the great majority of students, the use of the SBF framework allows effective reasoning about the structural, behavioral and functional roles within the system under study, in addition, this study's results have clear implications for the design of learning environments that can support learning about complex systems.

\section{REFERENCES}

[1] D. Chen, and W. Stroup, "General system theory: Toward a conceptual framework for science and technology education for all," Journal of Science Education and Technology, 2 (3), 447-459, 1993.

[2] M. Resnick, "Beyond the centralized mindset," Journal of the Learning Sciences, 5 (1), 1-22, 1996.

[3] K. J. Gilbert, and J. C. Boulter, (Eds.), "Developing models in science education," Dordrecht, Holland: Kluwer Academic Publishers, 2000.

[4] D. J. Gobert, and C. B. Buckley, "Introduction to model-based teaching and learning in science education," International Journal of Science Education, 22 (9), 891-894, 2000. 
[5] L. Louca, and C. Constantinou, "The use of computer-based microworlds for developing modeling skills in physical science: an example from light," International Journal of Science Education, 2003.

[6] C. Hmelo-Silver, and M. G. Pfeffer, "Comparing expert and novice understanding of a complex system from the perspective of structures, behaviours, and functions," Cognitive Science , 28 (1), 127-138, 2004.

[7] K. Hashem, and I. Arman, "Integration of ICT in mathematical understanding using modeling," International Journal of Computerand Information Technology, 2(2), 330-335, 2013.

[8] M. Resnick, and U. Wilensky, "Diving into Complexity: Developing probabilistic decentralized thinking through role-playing activities," Journal of Learning Sciences, 7 (2), 153-172, 1998.

[9] C. Yehezkel, M. Ben-Ari, and T. Dreyfus, "Computer architecture and mental models," ACM , 101-105, 2005.

[10] P. Blikstein, and U. Wilensky, "Less is more: agent-based simulation as a powerful learning tool in materials science," Proceedings of the IV International Joint Conference on AAMAS. Utrecht, Holland, 2005.

[11] K. Hashem, and D. Mioduser, "The Contribution of Learning by Modeling (LbM) to Students' Understanding of Complexity Concepts," International Journal of e-Education, e-Business, e-Management and $e$ Learning (IJEEEE) , 1 (2), 151-155, 2011.

[12] S. Levy, and U. Wilensky, "An analysis of student patterns of exploration with NetLogo models embedded in the connected chemistry environment," Proceedings of the annual meeting of the American Educational Research Association. Montreal, CA, 2005.

[13] M. Stieff, and U. Wilensky, "Connected chemistry-incorporating interactive simulations into the chemistry classroom," Journal of Science Education and Technology, 12 (3), 285-302, 2003.

[14] U. Wilensky, and M. Resnick, "Thinking in levels: A dynamic systems approach to making sense of the world," Journal of Science Education and Technology , 8 (1), 3-19, 1999.

[15] M. Jacobson, "Problem solving, cognition, and complex systems: Differences between experts and novices," Complexity, 6 (3), 41-49, 2001.

[16] M. Jacobson, and U. Wilensky, "Complex systems in education: scientific and educational importance and implications for the learning sciences," Journal of the Learning Sciences , 15 (1), 11-34, 2006.

[17] M. T. Chi, "Commonsense conceptions of emergent processes: why some misconceptions are robust?" The Journal of the Learning Sciences , 14 (2), 161-199, 2005.

[18] M. Resnick, "Changing the centralized mind. Cambridge," MA: MIT press, 1994.

[19] J. Gobert, "Harnessing technology to support on-line model building and peer collaboration," 2003.

[20] S. Tisue, and U. Wilensky, "NetLogo: A Simple Environment for Modeling Complexity," International Conferece on Complex Systems. Boston, 2004. 